Liu Shi-feng*, Shi Jia-min, Yang Xiao-kang, Cai Jun and Wang Qing-juan

\title{
High-Temperature Flow Behaviour and Constitutive Equations for a TC17 Titanium Alloy
}

https://doi.org/10.1515/htmp-2017-0145

Received October 16, 2017; accepted May 22, 2018

\begin{abstract}
In this study, the high-temperature deformation behaviour of a TC17 titanium alloy was investigated by isothermal hot compression tests in a wide range of temperatures $(973-1223 \mathrm{~K})$ and strain rates $\left(0.001-10 \mathrm{~s}^{-1}\right)$. Then, the constitutive equations of different phase regimes ( $\alpha+\beta$ and single $\beta$ phases) were developed on the basis of experimental stress-strain data. The influence of the strain has been incorporated in the constitutive equation by considering its effect on different material constants for the TC17 titanium alloy. Furthermore, the predictability of the developed constitutive equation was verified by the correlation coefficient and average absolute relative error. The results indicated that the obtained constitutive equations could predict the high-temperature flow stress of a TC17 titanium alloy with good correlation and generalization.
\end{abstract}

Keywords: TC17 titanium alloy, high-temperature flow behaviour, constitutive equation

\section{Introduction}

The TC17 (Ti-5Al-2Sn-2Zr-4Mo-4Cr) titanium alloy, as a "beta-rich" $\alpha+\beta$ titanium alloy, has an attractive combination of characteristics including high strength, excellent corrosion resistance, superior fracture toughness and deep hardenability, which make it an ideal candidate material for fan blades and compressor disks in aircraft engines [1]. This alloy has strength properties and creep resistance that are superior to those of Ti- $6 \mathrm{Al}-4 \mathrm{~V}$ alloys at the ultimate working temperature [2]. Therefore, considerable attention has been given in the scientific literature

*Corresponding author: Liu Shi-feng, School of Metallurgical Engineering, Xi'an University of Architecture and Technology, Xi'an 710055, China; State Key Laboratory of Powder Metallurgy, Central South University, Changsha 410083, China,

E-mail: liushifeng66@126.com

Shi Jia-min, Yang Xiao-kang, Cai Jun, Wang Qing-juan, School of Metallurgical Engineering, Xi'an University of Architecture and Technology, Xi'an 710055, China to investigations of the deformation behaviour of TC17 titanium alloys [3-7]. Moreover, the deformation behaviour of TC17 titanium alloys is sensitive to processing parameters such as deformation temperature and strain rate. Therefore, a thorough study regarding the high-temperature flow behaviour of TC17 titanium alloys is essential to properly choose the deformation parameters. However, the flow stress behaviour at high temperatures is very complex because the work hardening, dynamic softening (DS), dynamic recovery and dynamic recrystallization (DRX) are significantly influenced by the deformation parameters [8]. An effective method to describe flow behaviour of metal alloys is to develop suitable constitutive equations that can present the basic function between flow stress and processing parameters [9]. A considerable number of studies have been performed to propose a constitutive equation to predict the flow stress of various alloys [10-15].

Over the past few years, several constitutive models have been proposed to characterize the flow behaviour of both metals and alloys [1, 16, 17]. Among these models, the Johnson Cook (JC) [18] and Zerilli-Armstrong (ZA) [19] models have been successfully incorporated into the Finite Element (FE) to describe the thermal deformation behaviour of the alloy due to the small number of material constants involved and the small number of experiments required to evaluate the material constants. In addition, the sinusoidal hyperbola in the Arrhenius equation has been widely used to predict the high-temperature flow behaviour of the material [20-23]. Liang et al. [24] investigated the high-temperature compression and deformation behaviour of a powder metallurgy titanium alloy with a constitutive equation that considered strain compensation at a high temperature. The results show that the improved constitutive equation with a high temperature and a low strain rate has good predictability. Quan et al. [25] studied the DRX behaviour of the heatresistant alloy by regression analysis of the modified hyperbolic sine equation using an isothermal upsetting experiment.

Wang et al. [26] studied the dynamic globalization kinetics of lamellar microstructures during the hot 
working of a Ti17 alloy using isothermal compression tests. Liu et al. [1] investigated the constitutive model of a Ti17 titanium alloy with lamellar-type initial microstructure during hot deformation based on an orthogonal analysis. Ma et al. [27] used ANN and regression models to predict the flow stress of the lamellar initial microstructures of Ti17 titanium alloys in $\alpha$ and $\beta$ phase fields during thermal deformation. Wang et al. [28] characterized the microstructure of the adiabatic shearing band in a Ti17 alloy at high strain rates and elevated temperatures. Luo et al. [29] studied the flow behaviour and processing maps during the isothermal compression of a Ti17 alloy. Ma et al. [30] investigated the unsteady flow behaviour of a Ti17 alloy in an $\alpha+\beta$ phase field using a processing map. However, there are few involve the development of some constitutive equations to describe the hot deformation behaviour of the TC17 titanium alloys. Moreover, fewer research studies have focused on the model validation of Ti17 alloys at high temperatures and strain rates. Therefore, the main purpose of this investigation is to establish a suitable constitutive equation to predict the evaluated temperature flow stress of TC17 titanium alloys. To achieve this purpose in the current research, hot isothermal compression tests were carried out in a wide temperature range of 973-1223 K and a strain rate range of $0.001-10 \mathrm{~s}^{-1}$. Afterwards, the experimental stress-strain data were used to establish the constitutive equation. Finally, the developed constitutive equations were validated by the correlation coefficient $(R)$ and average absolute relative error ( $A A R E)$.

\section{Experimental procedure}

A micrograph of the TC17 titanium alloy that was employed in this work is shown in Figure 1. The chemical composition of the TC17 titanium alloy is provided in Table 1. From this figure, the results indicate that the microstructure of the as-received TC17 titanium alloy consists of an equiaxed primary $\alpha$ phase (hexagonal close-packed) and an intergranular $\beta$ phase (bodycentred cubic).

The alloy was machined into cylindrical specimens with a diameter of $10 \mathrm{~mm}$ and a height of $15 \mathrm{~mm}$ so that it had an excellent (N6 grade) surface for compression testing to eliminate any surface effects on the flow behaviour. To obtain a heat balance, each specimen was heated to the deformation temperature at a rate of $10 \mathrm{~K} / \mathrm{s}$ and held for $5 \mathrm{~min}$ at isothermal conditions prior to isothermal compression for eliminating thermal

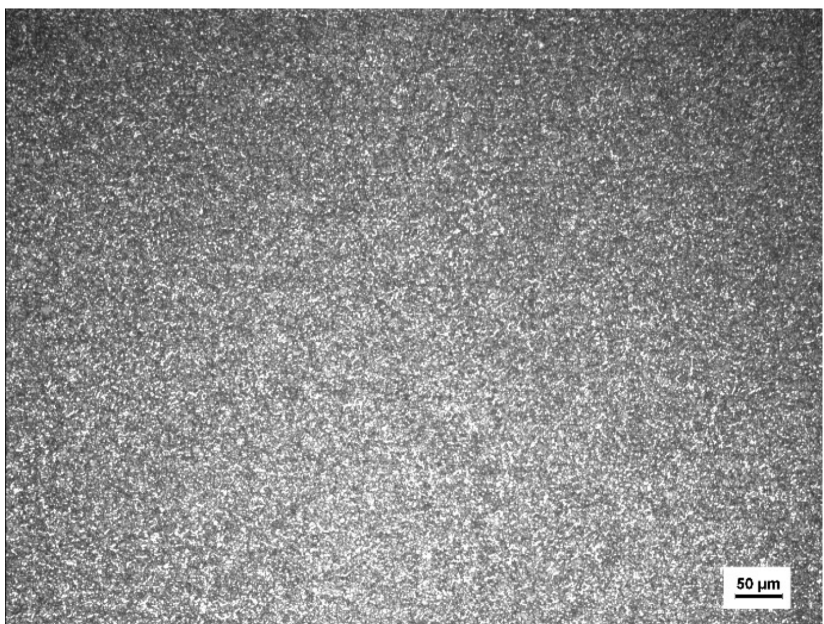

Figure 1: Micrograph of the as received TC17 titanium alloy.

gradients. Then, isothermal compression tests were carried out on a Gleeble-3500 thermal mechanical simulator at strain rates of $0.001,0.01,0.1,1$ and $10 \mathrm{~s}^{-1}$ and deformation temperatures of $973,1023,1073,1123,1183$ and $1223 \mathrm{~K}$. The final deformation degree was $60 \%$, which is similar to that of Ma et al. [30] After deformation, the specimens were quenched in water, and the strain-stress curves were recorded automatically in isothermal compression. The $\beta$ transus temperature of the TC17 titanium alloy is approximately $1168 \mathrm{~K}$. Therefore, four and two temperatures were chosen in the $\alpha+\beta$ and single $\beta$ phase regions, respectively.

\section{Results and discussion}

\section{Flow stress}

The true stress-strain curves that were obtained from the hot compression tests of the TC17 titanium alloy are shown in Figure 2. These data show that the deformation temperature and strain rate influence the flow stress. The flow stress increases with an increase in the strain rate at a certain temperature and decreases monotonically with an increase in the deformation temperature at a particular strain rate. Moreover, the results in Figure 2 indicate that the TC17 titanium alloy shows obvious thermal softening and strain rate hardening. At the initial stage of deformation, the flow stress rapidly increases with an increase in strain because the dislocation generation, multiplication and intersect are significant with an increase in deformation. Thereafter, with an increase in 
Table 1: Chemical composition (in wt \%) of TC17 titanium alloy.

\begin{tabular}{lcccccccccc}
\hline Al & Cr & Mo & Sn & Zr & Fe & C & N & H & 0 & Ti \\
\hline 5.15 & 4.01 & 4.02 & 2.16 & 2.09 & 0.096 & 0.009 & 0.008 & 0.006 & 0.12 & Bal. \\
\hline
\end{tabular}
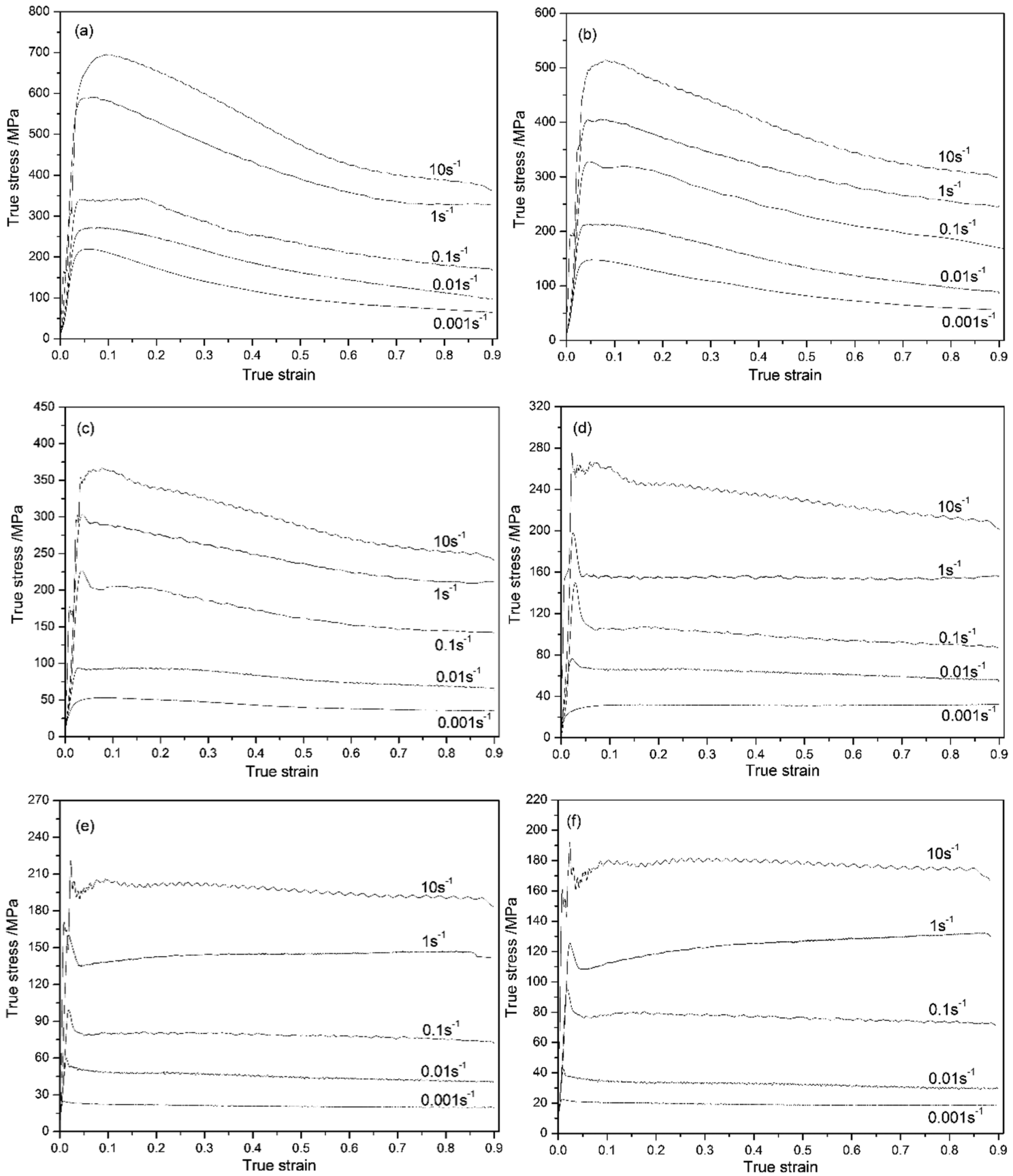

Figure 2: Flow stress curves of as-received Ti-6Al-4V alloy at temperatures of (a) 973K; (b) 1023; (c) 1073K; (d) 1123K; (e) 1173K; (f) 1223K.

deformation, the flow stress reaches a peak at a critical strain. At lower deformation temperatures, such as 973 , 1023 and 1073K, the flow stress then continuously decreases to a steady value owing to DS, showing a noticeable softening phenomenon. However, above the $\beta$ rotor temperature (1173K and $1223 \mathrm{~K}$ ), the flow stress 
first exhibits a decrease and then increases to a stable flow behaviour (Figure 2(e) and (f)). A similar phenomenon has also been reported for the TC18 alloy [31].

\section{Constitutive equation}

The Arrhenius constitutive equation has been widely used to represent the relationship between the flow stress and strain rate as well as deformation temperatures. The effects of strain rate and deformation temperature on the metal flow stress can be expressed by the ZenerHolloman parameter $(Z)$ in an exponent type equation as follows:

$$
\begin{gathered}
Z=\dot{\varepsilon} \exp \left(\frac{Q}{R T}\right) \\
\dot{\varepsilon}=A F(\sigma) \exp \left(-\frac{Q}{R T}\right) \\
F(\sigma)=\left\{\begin{array}{lll}
\sigma^{n^{\prime}} & \alpha \sigma<0.8 \\
\exp (\beta \sigma) & \alpha \sigma>1.2 \\
{[\sinh (\alpha \sigma)]^{n}} & \text { for } \quad \text { all } \quad \sigma \\
\end{array}\right.
\end{gathered}
$$

where $\dot{\varepsilon}$ is the strain rate $\left(\mathrm{s}^{-1}\right), R$ is the universal gas constant $\left(8.31 \mathrm{~J} \cdot \mathrm{mol}^{-1} \cdot \mathrm{K}^{-1}\right), T$ is the absolute temperature $(\mathrm{K})$, and $Q$ is the activation energy of hot deformation $\left(\mathrm{J} \cdot \mathrm{mol}^{-1}\right) . A, n^{\prime}, \beta, \alpha$ and $n$ are the materials constants, and $\alpha=\beta / n^{\prime}$.

At a certain deformation temperature for both lower and higher stress levels, substituting eqs (1) and (3) into eqs (2), (4) and (5) can be obtained as follows:
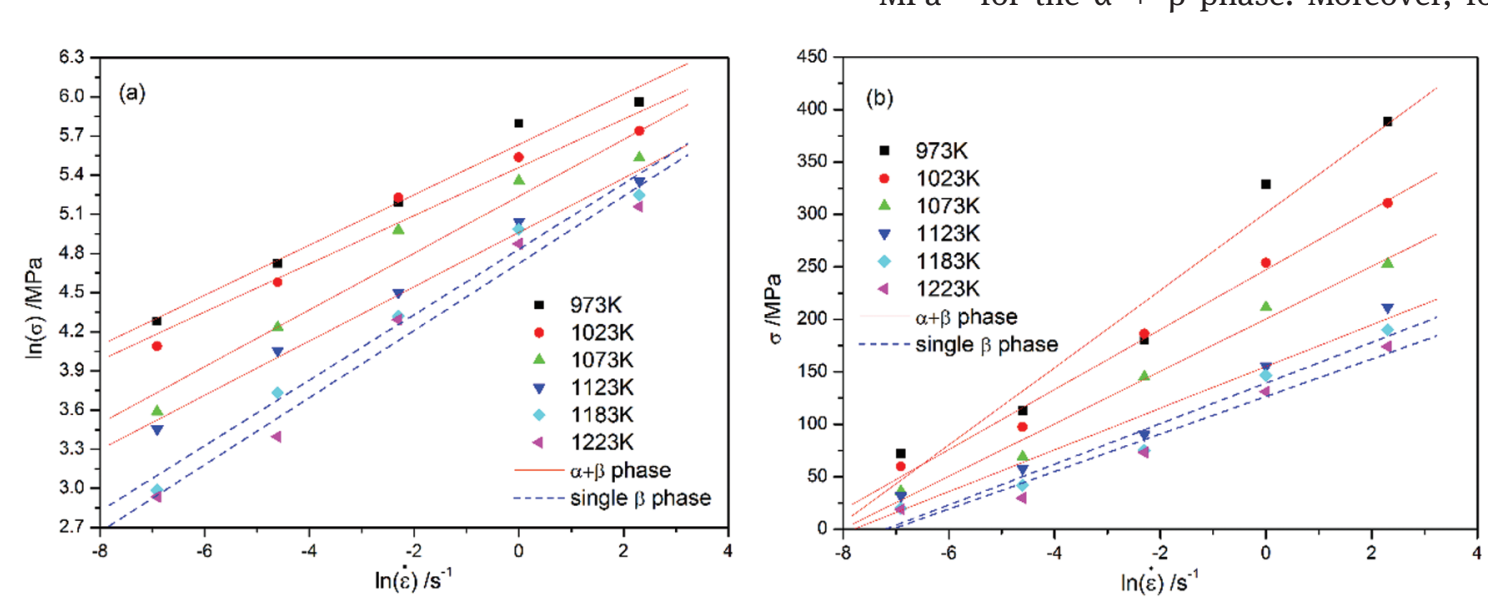

Figure 3: Relationship between (a) $\ln (\sigma)$ and $\ln (\dot{\varepsilon})$ (b) $\sigma$ and $\ln (\dot{\varepsilon})$ at the strain of 0.8 .

$$
\begin{gathered}
\dot{\varepsilon}=B \sigma^{n^{\prime}} \\
\dot{\varepsilon}=C \exp (\beta \sigma)
\end{gathered}
$$

where $B$ and $C$ are the material constants, which are independent of the deformed temperatures. Then, taking the logarithm of both sides of eqs (4) and (5), the following equations can be obtained:

$$
\begin{gathered}
\ln (\sigma)=\frac{1}{n^{\prime}} \ln (\dot{\varepsilon})-\frac{1}{n^{\prime}} \ln (B) \\
\sigma=\frac{1}{\beta} \ln (\dot{\varepsilon})-\frac{1}{\beta} \ln (C)
\end{gathered}
$$

Therefore, the values of $n^{\prime}$ and $\beta$ can be acquired by the slope of the lines in the $\ln (\sigma)-\ln (\dot{\varepsilon})$ as well as $\sigma-\ln (\dot{\varepsilon})$ plots, as shown in Figure 3(a) and (b).

In Figure 3, the slopes of the lines exhibit an obvious difference in the $\alpha+\beta$ and single $\beta$ phases. Figure 4 shows the microstructures of the TC17 titanium

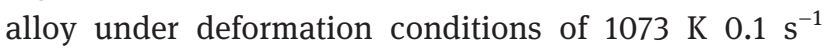

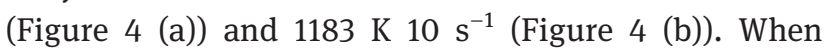
the deformation temperature was lower than the $\beta$ transus temperature, the microstructure of the TC17 titanium alloy consisted of an $\alpha+\beta$ phase, as shown in Figure $4(\mathrm{a})$. When the deformation temperature was above the $\beta$ transus temperature, the final microstructure only consisted of $\beta$ grains after deformation, as shown in Figure 4(b). Therefore, the material constants should be calculated for the $\alpha+\beta$ and single $\beta$ phases separately. As the flow stress began to reach a stable stage, the strain of 0.8 was taken as the example to introduce the solution process of the material constants. The mean values of the slopes were taken as the values of $n^{\prime}$ and $\beta$ that were estimated to be 4.9812 and 0.03625 $\mathrm{MPa}^{-1}$ for the $\alpha+\beta$ phase. Moreover, for the single $\beta$ 

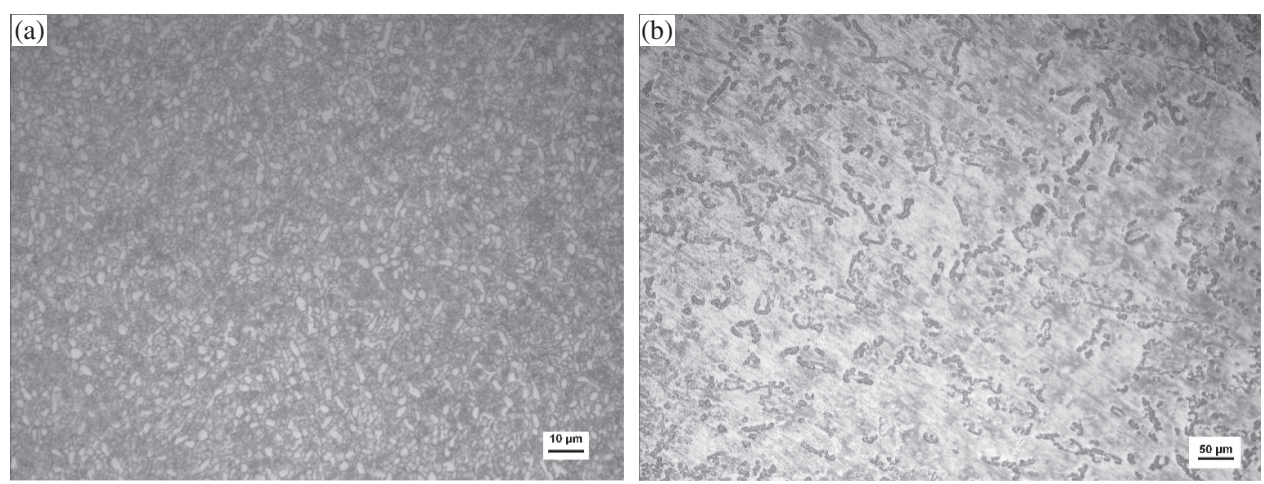

Figure 4: Microstructure of TC17 titanium alloy under the deformation condition of (a) $1073 \mathrm{~K}, 0.1 \mathrm{~s}^{-1}$, and (b) $1183 \mathrm{~K}, 10 \mathrm{~s}^{-1}$.

phase, the mean values of $n^{\prime}$ and $\beta$ were calculated to be 3.9341 and $0.05371 \mathrm{MPa}^{-1}$, respectively. Therefore, the values of $\alpha$ were calculated to be 0.007278 for the $\alpha+\beta$ phase and 0.01367 for the single $\beta$ phase.

For low as well as high stress levels, eq. (2) can be written as follows:

$$
\dot{\varepsilon}=A[\sinh (a \sigma)]^{n} \exp \left(-\frac{Q}{R T}\right)
$$

Then, taking the logarithm of both sides of eqs (8), (9) can be obtained:

$$
\ln [\sinh (\alpha \sigma)]=\frac{\ln \dot{\varepsilon}}{n}+\frac{Q}{n R T}-\frac{\ln A}{n}
$$

For a particular temperature, differentiating eq. (10) gives the following:

$$
\frac{1}{n}=\frac{d\{\ln [\sinh (\alpha \sigma)]\}}{d(\ln \dot{\varepsilon})}
$$

Therefore, the value of $n$ can be obtained from the slopes of the lines of $\ln [\sinh (\alpha \sigma)]-\ln \dot{\varepsilon}$, as illustrated in Figure 5 . The value of $n$ is calculated by averaging the values of $n$ at various deformation temperatures. Therefore, the values $n$ for the $\alpha+\beta$ and single $\beta$ phases were estimated to be 3.6592 and 2.8490 , respectively.

For a given strain rate, differentiating eq. (9) yields the following:

$$
Q=R n \frac{d\{\ln [\sinh (\alpha \sigma)]\}}{d(1 / T)}
$$

The values of $Q$ for the $\alpha+\beta$ and single $\beta$ phases can be acquired from the slopes in the plot of $\ln [\sinh (a \sigma)]-1 / T$, as shown in Figure 6. The value of $Q$ can be calculated by averaging the $Q$ values at various strain rates. At a true strain of 0.8 , the $Q$-values were determined to be 234.06

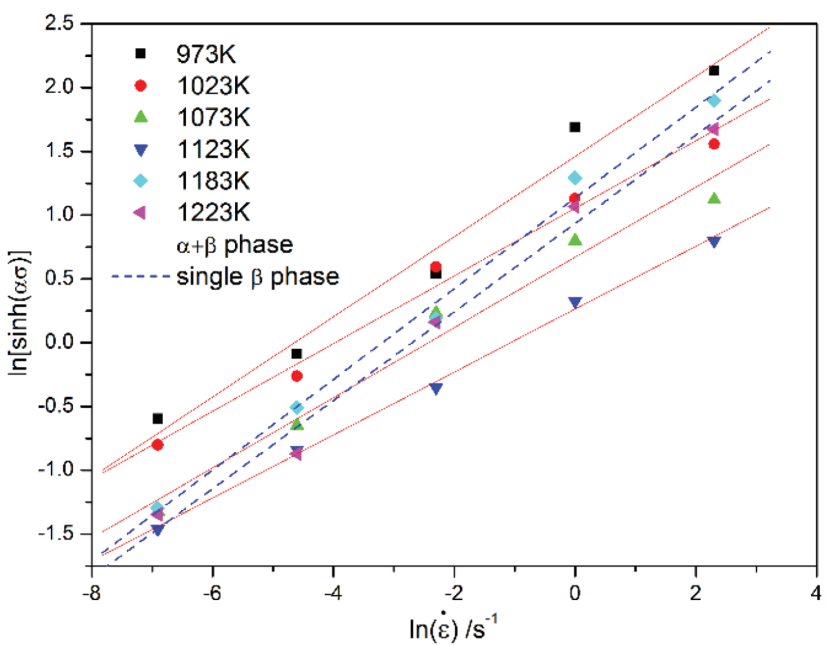

Figure 5: Relationship between $\ln [\sinh (\alpha \sigma)]$ and $\ln (\dot{\varepsilon})$ at the strain of 0.8 .

$\mathrm{kJ} / \mathrm{mol}$ for the $\alpha+\beta$ phase and $154.02 \mathrm{~kJ} / \mathrm{mol}$ for the single $\beta$ phase. In previous research, Ma et al. [27] estimated that the activation energy at a strain of 0.7 was $320.422 \mathrm{~kJ} / \mathrm{mol}$ for the $\alpha+\beta$ phase. Li et al. [32] inferred that the average value of the activation energy at a strain of 0.5 was $392.3 \mathrm{~kJ} / \mathrm{mol}$ for the $\alpha+\beta$ phase. Li et al. [31] found that the average value of the activation energy was $187.25 \mathrm{~kJ} / \mathrm{mol}$ for the $\beta$ phase. The activation energy in this work is lower than those obtained by $\mathrm{Ma}$ et al. and $\mathrm{Li}$ et al. This phenomenon could be due to the initial microstructure and heat treatment conditions in the alloy studied [33]. Furthermore, the values of $A$ at a certain strain can be derived from the intercept in Figure 5 .

Therefore, taking $\alpha, n, Q$ and $A$ into eq. (8), the constitutive equation of the TC17 titanium alloy can be obtained. However, the influence of strain on the flow stress has been neglected in eq. (8).Nevertheless, strain 

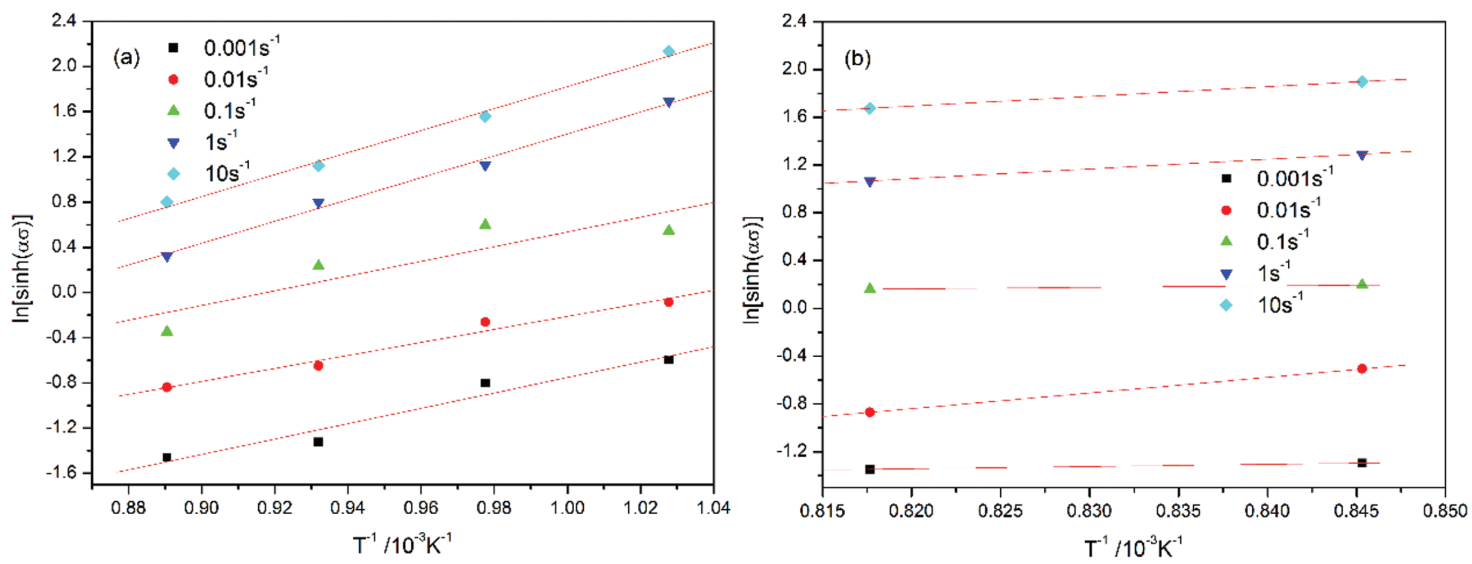

Figure 6: Relationship between $\ln [\sinh (\alpha \sigma)]$ and $1 / T$ for (a) $\alpha+\beta$ phase (b) $\beta$ phase.

has an obvious influence on the flow stress, particularly at lower temperatures, as shown in Figure 2 (a) and (b). Moreover, the effect of strain on the material constants $\alpha$, $n, Q$ and $\ln A$ is significant over the entire strain range, as given in Figure 7. Therefore, the compensation of strain on the material constants should be considered to acquire an accurate constitutive equation. Clearly, the values of $\alpha$ tend to increase monotonically with an
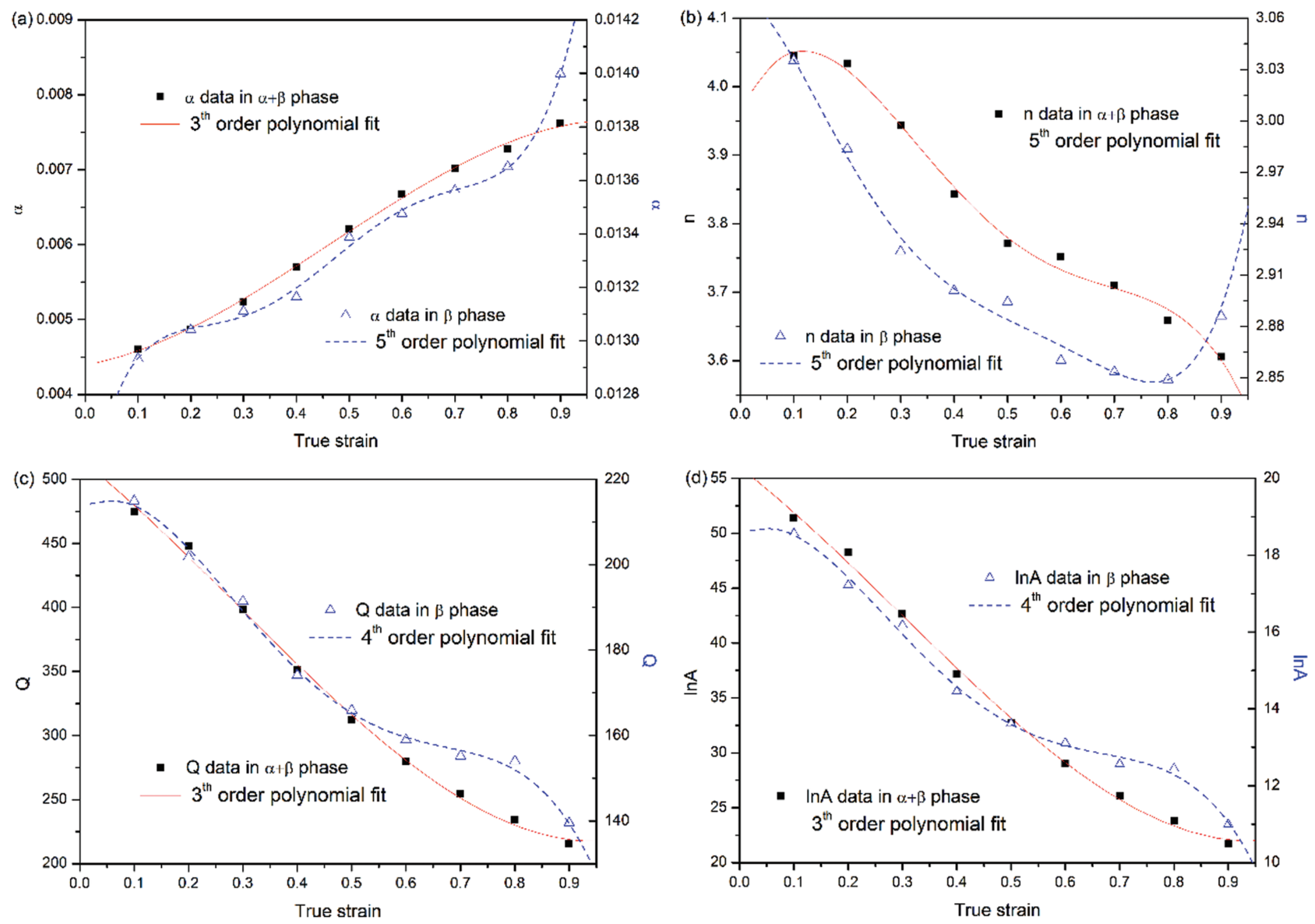

Figure 7: Variation of (a) $\alpha$, (b) $n$, (c) $Q$ and (d) $\ln A$ with true strain. 
increase in strain for both the $\alpha+\beta$ and single $\beta$ phases, as given in Figure $7(\mathrm{a})$. The $n$ values decrease with an increase in strain in the $\alpha+\beta$ phase, whereas they decrease to the minimum value at a strain of 0.8 and then increase in the single $\beta$ phase, as shown in Figure 7 (b). The values of $Q$ and $\ln A$ decrease monotonically with an increase in strain for both the $\alpha+\beta$ and single $\beta$ phases, as illustrated in Figure 7 (c) and (d).

In this study, the influence of strain on the flow stress in the constitutive equations was incorporated by assuming that the material constants $(\alpha, n, Q$ and $\ln A)$ are a polynomial function of strain $[34,35]$. The values of the material constants $(\alpha, n, Q$ and $\ln A)$ were evaluated in the strain range of $0.1 \sim 0.9$ with an interval of 0.1. The order of the polynomial varied from 1 to 9 . On the basis of the good fitting correlation and accuracy, a third-order polynomial was found to represent the influence of strain on $\alpha, Q$ and $\ln A$ for the $\alpha+\beta$ phase. A fifth-order polynomial was employed to express the effect of strain on $n$ in the $\alpha+\beta$ phase and $\alpha$ as well as $n$ in the single $\beta$ phase, as shown in eq. (12). Meanwhile, a fourth-order polynomial was used to represent the influence of strain on $Q$ and $\ln A$ for the single $\beta$ phase. The values of polynomial fitting coefficients of $\alpha, n, Q$ and $\ln A$ of the TC17 titanium alloy for the $\alpha+\beta$ and single $\beta$ phases are given in Tables 2 and 3, respectively.

Table 2: Coefficients of the polynomial for $\alpha, n, Q$, and $\ln A$ for $\alpha+\beta$ phase.

\begin{tabular}{llll}
\hline $\boldsymbol{\alpha}$ & $\boldsymbol{n}$ & $\boldsymbol{Q}$ & $\ln \boldsymbol{}$ \\
\hline $\mathrm{C}_{0}=0.00439$ & $\mathrm{D}_{0}=3.9612$ & $\mathrm{E}_{0}=516.275$ & $\mathrm{~F}_{0}=-0.67266$ \\
$\mathrm{C}_{1}=0.00119$ & $\mathrm{D}_{1}=1.7027$ & $\mathrm{E}_{1}=-340.729$ & $\mathrm{~F}_{1}=-38.4473$ \\
$\mathrm{C}_{2}=0.00742$ & $\mathrm{D}_{2}=-9.5302$ & $\mathrm{E}_{2}=-283.108$ & $\mathrm{~F}_{2}=-33.86268$ \\
$\mathrm{C}_{3}=-0.00534$ & $\mathrm{D}_{3}=14.3053$ & $\mathrm{E}_{3}=327.306$ & $\mathrm{~F}_{3}=38.49652$ \\
& $\mathrm{D}_{4}=-7.0152$ & & \\
\hline
\end{tabular}

Table 3: Coefficients of the polynomial for $\alpha, n, Q$, and $\ln A$ for single $\beta$ phase.

\begin{tabular}{llll}
\hline $\boldsymbol{\alpha}$ & $\boldsymbol{n}$ & $\boldsymbol{Q}$ & $\ln \boldsymbol{A}$ \\
\hline $\mathrm{C}_{0}=0.0124$ & $\mathrm{D}_{0}=3.0769$ & $\mathrm{E}_{0}=212.4888$ & $\mathrm{~F}_{0}=18.5124$ \\
$\mathrm{C}_{1}=0.0093$ & $\mathrm{D}_{1}=-0.1485$ & $\mathrm{E}_{1}=111.0179$ & $\mathrm{~F}_{1}=8.5369$ \\
$\mathrm{C}_{2}=-0.0523$ & $\mathrm{D}_{2}=-3.6811$ & $\mathrm{E}_{2}=-1105.0489$ & $\mathrm{~F}_{2}=-101.4897$ \\
$\mathrm{C}_{3}=0.1387$ & $\mathrm{D}_{3}=12.8288$ & $\mathrm{E}_{3}=1880.7483$ & $\mathrm{~F}_{3}=176.4400$ \\
$\mathrm{C}_{4}=-0.1624$ & $\mathrm{D}_{4}=-16.6305$ & $\mathrm{E}_{4}=-988.1030$ & $\mathrm{~F}_{4}=-93.8398$ \\
$\mathrm{C}_{5}=0.0694$ & $\mathrm{D}_{5}=7.5941$ & & \\
\hline
\end{tabular}

$$
\begin{aligned}
& \alpha=C_{0}+C_{1} \varepsilon+C_{2} \varepsilon^{2}+C_{3} \varepsilon^{3}+C_{4} \varepsilon^{4}+C_{5} \varepsilon^{5} \\
& n=D_{0}+D_{1} \varepsilon+D_{2} \varepsilon^{2}+D_{3} \varepsilon^{3}+D_{4} \varepsilon^{4}+D_{5} \varepsilon^{5} \\
& Q=E_{0}+E_{1} \varepsilon+E_{2} \varepsilon^{2}+E_{3} \varepsilon^{3}+E_{4} \varepsilon^{4}+E_{5} \varepsilon^{5} \\
& \ln A=F_{0}+F_{1} \varepsilon+F_{2} \varepsilon^{2}+F_{3} \varepsilon^{3}+F_{4} \varepsilon^{4}+F_{5} \varepsilon^{5}
\end{aligned}
$$

Once the material constants are obtained, the flow stress at a certain strain can be predicted from following equation (considering the eqs (1) and (8)). The constitutive equation that relates the flow stress and ZenerHolloman parameter can be written as follows by using the expression of a hyperbolic sine function.

$$
\sigma=\frac{1}{\alpha} \ln \left\{\left(\frac{Z}{A}\right)^{\frac{1}{n}}+\left[\left(\frac{Z}{A}\right)^{\frac{2}{n}}+1\right]^{\frac{1}{2}}\right\}
$$

\section{Verification of the developed constitutive equations}

The obtained constitutive equations in consideration of strain were assessed by comparing the experimental and predicted flow stress, as shown in Figure 8. The predicted flow stress values from the developed constitutive equations can track the experimental data of the TC17 titanium alloy throughout the entire temperature range. Similar prediction accuracy has also been obtained in the single $\beta$ phase region. Some variation between experimental and computed flow stress data can be observed only under some deformation conditions in the $\alpha+\beta$ phase region (Figure 8 (a) and (d)). Under some deformation conditions in the single $\beta$ phase region (i.e. at $1183 \mathrm{~K}$ in $1 \mathrm{~s}^{-1}$ and at $1223 \mathrm{~K}$ in $1 \mathrm{~s}^{-1}$ ), there may be contradictions between experimental flow and calculated flow stress data (Figure 8 (e) and (f)). The main reason of the difference may contribute to the fact that the response of flow behavior of materials at high temperatures is highly nonlinear [36]. Many factors that affect flow stress are nonlinear, which makes the constitutive equation predict the flow stress is less accurate, and the application range is limited [37]. Possibly, the fitting of material constants leads to the variation between experimental and computed flow stress data. For example, experimental data in Figure 5 appear some deviation. Therefore, since the value of $\beta$ is determined using eq. (7), some errors may be introduced and ultimately affect the accuracy of the constitutive equation. Further research needs to be done to draw a firm conclusion.

The predictability of the developed constitutive equations of the TC17 titanium alloy was further 

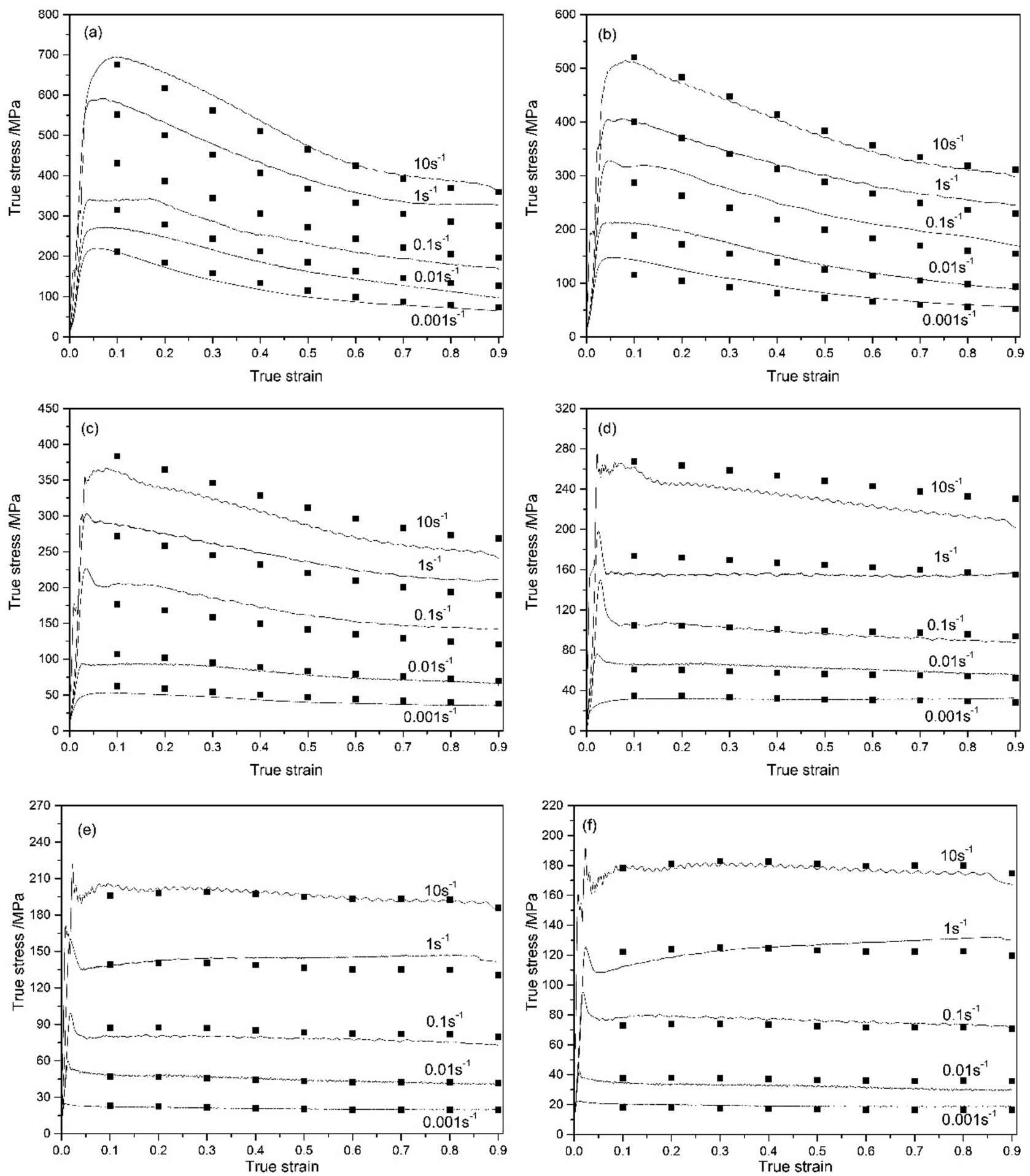

Figure 8: Comparison between the experimental and predicted flow stress at the temperature (a) 973K; (b) 1023K; (c) 1073K; (d) 1123K; (e) $1183 \mathrm{~K}$; (f) $1223 \mathrm{~K}$. 
quantified in terms of standard statistical parameters such as the $R$ and $A A R E$, which are expressed as follows:

$$
\begin{gathered}
R=\frac{\sum_{i=1}^{N}\left(E_{i}-\bar{E}\right)\left(P_{i}-\bar{P}\right)}{\sqrt{\sum_{i=1}^{N}\left(E_{i}-\bar{E}\right)^{2} \sum_{i=1}^{N}\left(P_{i}-\bar{P}\right)^{2}}} \\
\operatorname{AARE}(\%)=\frac{1}{N} \sum_{i=1}^{N}\left|\frac{E_{i}-P_{i} \mid}{E_{i}}\right| \times 100
\end{gathered}
$$

where $E$ is the value of the experimental flow stress and $P$ is the predicted flow stress value calculated by the developed constitutive equation. $\bar{E}$ and $\bar{P}$ are the mean values of $E$ and $P$, respectively. $N$ is the total number of data employed in this research. $R$ is a commonly employed statistical parameter and can provide information regarding the strength of the linear relationship between the experimental and predicted data [38]. Sometimes, a higher value of $R$ may not necessarily reflect better performance because the tendency of the equation may be biased towards higher or lower values [39]. However, the $A A R E$ was obtained from a term-by-term comparison of the relative error. Therefore, the $A A R E$ is an unbiased statistical parameter for determining the predictability of the equation [40]. Figure 9 provides the correlation between the experimental and predicted flow stress data from the developed constitutive equations. The findings in Figure 9 demonstrate that the values of $R$ and $A A R E$ are 0.988 and $8.84 \%$, respectively, in the $\alpha+\beta$ phase region and 0.997 and $5.56 \%$, respectively, in the single $\beta$ phase region. These results reflect the good prediction capabilities of the proposed constitutive equation with the consideration of strain.

\section{Conclusions}

The high-temperature deformation behaviour of a TC17 titanium alloy was investigated by performing hot compression tests in the temperature range of $973-1223 \mathrm{~K}$ and the strain rate range of $0.001-10 \mathrm{~s}^{-1}$. Based on this study, the conclusions are presented as follows:

(1) The flow stress of the TC17 titanium alloy at a high temperature is significantly influenced by deformation temperature and strain rate. The flow stress decreases with an increasing deformation temperature and a decreasing strain rate. The strain influenced the flow stress in the $\alpha+\beta$ phase but had little effect in the single $\beta$ phase.

(2) The influence of strain in the constitutive equations of the TC17 titanium alloy including the $\alpha+\beta$ and single $\beta$ phases was incorporated by considering the effect of strain on the material constants $\alpha, n, Q$ and $\ln A$. A third-order polynomial suitably represented the influence of strain on the material constants $\alpha, Q$ and $\ln A$ for the $\alpha+\beta$ phase. A fourth-order polynomial was observed to adequately express the
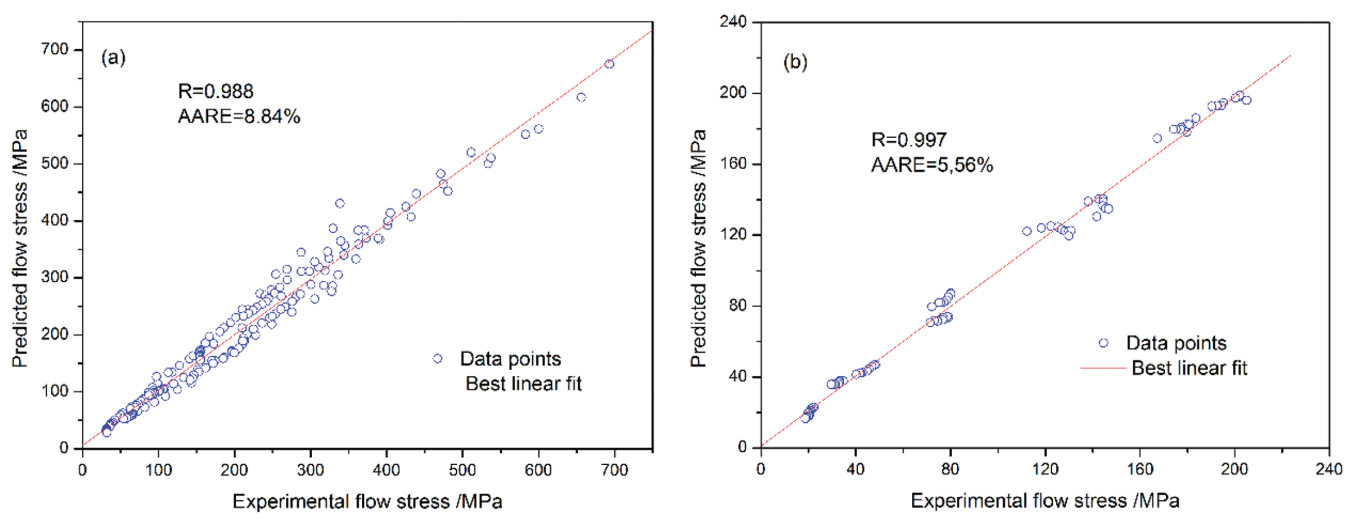

Figure 9: Correlation between the experimental and predicted flow stress data from the developed constitutive equations (a) $\alpha+\beta$ phase; (b) single $\beta$ phase. 
influence of strain on $Q$ and $\ln A$ for the single $\beta$ phase, and a fifth-order polynomial was employed to express the effect of strain on $n$ in the $\alpha+\beta$ phase and $\alpha$ as well as $n$ in the single $\beta$ phase with good correlation and generalization.

(3) The developed constitutive equation that compensates for strain can precisely predict the flow stress under most deformation conditions in both the $\alpha+$ $\beta$ and single $\beta$ phases. It is only under certain deformation conditions in the $\alpha+\beta$ phase that some deviations can be observed between the experimental and calculated flow stress data.

(4) The predictability of the developed constitutive equation was evaluated by the $R$ and $A A R E$. The values of $R$ and $A A R E$ were found to be 0.988 and $8.84 \%$, respectively, in the $\alpha+\beta$ phase and 0.997 and $5.56 \%$, respectively, in the single $\beta$ phase. The results reflected the good prediction capabilities of the proposed constitutive equations.

Acknowledgements: The authors gratefully acknowledge the financial support received from the National Natural Science Foundation of China (51874225), Innovation Foundation of Western Materials (XBCL-319), Planned Scientific Research Project of Education Department of Shaanxi Provincial Government (15JS056), Industrial Science and Technique Key Project of Shaanxi Province (2016GY-207), Project of International Cooperation and Exchange of Shaanxi Provincial (2016KW-054), and Province Natural Science Foundation of Shaanxi (2014JM6230)

\section{References}

[1] J.L. Liu, W.D. Zeng, Y.J. Lai and Z.Q. Jia, Mater. Sci. Eng. A., 597 (2014) 387-394.

[2] B.L. Wang, X. Ai, Z.Q. Liu and J.G. Liu, J. Wuhan Univ. Technol.Mat. Sci. Edit., 30 (2015) 611-616.

[3] Y.G. Liu, H.M. Li and M.Q. Li, Mater. Des., 65 (2015) 120-126.

[4] X. Ma, W.D. Zeng, K.X. Wang, Y.J. Lai and Y.G. Zhou, Mater. Sci. Eng. A., 550 (2012) 131-137.

[5] Y.D. Wang, H. Zheng, K. Tang, H.X. Li and S.L. Gong, Opt. Las. Technol., 82 (2016) 1-9.

[6] S.B. Jiao, C. Gao, L. Cheng, X.W. Li and Y. Feng, Mater. Eng. Per., 25 (2016) 1085-1093.

[7] Z.W. Cao, H.Y. Xu, S.K. Zou and Z.G. Che, Chinese J. Aero., 25 (2012) 650-656.

[8] M.H. Wang, G.T. Wang and R.J. Wang, Cent. South Univ., 23 (2016) 1863-1872.

[9] Y. Sun, W.H. Ye and L.X. Hu, Mater. Eng. Per., 25 (2016) $1621-1630$
[10] B. Li, Q.L. Pan, C. Li, Z.Y. Zhang and Z.M. Yin, Cent. Sou. Univ., 20 (2013) 2939-2946.

[11] B.J. Yu, X.J. Guan, L.J. Wang, J. Zhao, Q.Q. Liu and Y. Cao, Cent. Sou. Univ. Technol., 18 (2011) 36-41.

[12] X.B. Liu, G.L. Wu and C.Y. Zhou, J. Cent. Sou. Univ., 23 (2016) 3065-3071.

[13] G. Wu, C. Zhou and X. Liu, J. Cent. South Univ., 23 (2016) 1007-1014.

[14] J. Cai, X.L. Zhang, K.S. Wang and C.P. Miao, High Temp. Mater. Pro., 35 (2016) 1037-1045.

[15] A. Ghatak and P.S. Robi, Mater. Sci. Eng. A., 648 (2015) 418-427.

[16] H.Y. Li, X.F. Wang, D.D. Wei, J.D. Hu and Y.H. Li, Mater. Sci. Eng. A., 536 (2012) 216-222.

[17] J. Cai, K.S. Wang, P. Zhai, F.G. Li and J. Yang, Mater. Eng. Per., 24 (2015) 32-44.

[18] G.R. Johnson and W.H. Cook, Eng. Fra. Mech., 21 (1985) 31-48.

[19] C.M. Sellars and W.J. McTegart, Acta Metal., 14 (1966) $1136-1138$.

[20] A. Abbasi-Bani, A. Zarei-Hanzaki, M.H. Pishbin and N. Haghdadi, Mater. Mech., 71 (2014) 52-61.

[21] H.Y. Li, Y.H. Li, X.F. Wang, J.J. Liu and Y. Wu, Mater. Des., 49 (2013) 493-501.

[22] J. Li, F.G. Li, J. Cai, R.T. Wang, Z.W. Yuan and G.L. Ji, Comp. Mater. Sci., 71 (2013) 56-65.

[23] Y.C. Lin and X.M. Chen, Mater. Des., 32 (2011) 1733-1759.

[24] X.P. Liang, Y. Liu, H.Z. Li, C.X. Zhou and G.F. Xu, Mater. Des., 37 (2012) 40-47.

[25] G.Z. Quan, A. Mao, G.C. Liu, J.T. Liang and D.S. Wu, Mater. Des., 52 (2013) 98-107.

[26] K.X. Wang, W.D. Zeng, Y.Q. Zhao and Y.J. Lai, Mater. Sci. Eng. A., 527 (2010) 2559-2566.

[27] X. Ma, W.D. Zeng, Y. Sun, K.X. Wang, Y.J. Lai and Y.G. Zhou, Mater. Sci. Eng. A., 538 (2012) 182-189.

[28] Y.B. Wang, W.D. Zeng, X. Sun and J.W. Xu, Mater. Sci. Eng. A., 677 (2016) 325-331.

[29] J. Luo, L. Li and M.Q. Li, Mater. Sci. Eng. A., 606 (2014) 165-174.

[30] X. Ma, W.D. Zeng and K.X. Wang, Mater. Sci. Eng. A., 550 (2012) 131-137.

[31] C.W. Li, H. Xie, X.N. Mao, P.S. Zhang and Z.M. Hou, Rare Metal Mater. Eng., 46 (2017) 0326-0332.

[32] L. Li and M.Q. Li, Mater. Sci. Eng. A., 698 (2017) 302-312.

[33] Y.T. Wu, Y.C. Liu, C. Li, X.C. Xia, Y. Huang, H.J. Li and H.P. Wang, J. Alloy Compd., 712 (2017) 687-695.

[34] D. Samantaray, S. Mandal and A.K. Bhaduri, Mater. Des., 31 (2010) 981-984.

[35] A. Abbasi-Bani, A. Zarei-Hanzaki, M.H. Pishbin and N. Haghdadi, Mater. Mech., 71 (2014) 52-61.

[36] J. Cai, K.S. Wang, C.P. Miao et al., Mater. Des., 65 (2015) 272-279.

[37] H.Y. Li, X.F. Wang, D.D. Wei et al., Mater. Sci. Eng. A., 536 (2012) 216-222.

[38] M.P. Phaniraj and A.K. Lahiri, J. Mater. Pro. Technol., 141 (2003) 219-227.

[39] J. Cai, F.G. Li, T.Y. Liu, B. Chen and M. He, Mater. Des., 32 (2011) 1144-1151.

[40] S. Mandal, P.V. Sivaprasad, S. Venugopal and K.P.N. Murthy, Appl. Soft Comp., 9 (2009) 237-244. 